\title{
Subgap spectrum for an interacting hybrid superconducting quantum dot
}

\author{
Jong Soo Lim $\odot^{1}$ and Rosa López ${ }^{2}$ \\ ${ }^{1}$ School of Physics, Korea Institute for Advanced Study, Seoul 130-722, Korea \\ ${ }^{2}$ Institut de Física Interdisciplinària i de Sistemes Complexos IFISC (CSIC-UIB), E-07122 Palma de Mallorca, Spain
}

(Received 9 April 2020; revised manuscript received 11 May 2020; accepted 5 June 2020; published 22 June 2020)

\begin{abstract}
We examine the formation of Andreev bound states in a metal-quantum-dot-BCS superconductor system in the Coulomb blockade regime and the Kondo regime. We propose to use the so-called Hubbard-I decoupling scheme in the context of the equation-of-motion technique to provide a proper physical description for the Coulomb blockade regime. Here, we find the appearance of anticrossings in the subgap spectrum due to the prevalence of even parity states independently on the dot gate position. On the contrary, for the Kondo regime the subgap structure exhibits parity crossings between the alternance of singlet and doublet states. Besides, we show how the nature of the ground state in this regime can be distinguished from evolution of the Andreev states in the presence of a magnetic field.
\end{abstract}

DOI: 10.1103/PhysRevB.101.245427

\section{INTRODUCTION}

An unpaired electron residing in a quantum dot (QD) which is in close proximity with a superconductor offers a plethora of nontrivial phenomena [1,2]. The richness of its physical behavior has been probed in a great variety of setups such as magnetic adatoms [3], carbon nanotube QDs [4-11], nanowire QDs [12-15], self-assembled QDs [16-18], and graphene QDs [19].

In the system under consideration, a QD is tunnel coupled to a superconductor $(\mathrm{S})$ and a normal $(\mathrm{N})$ reservoir. Here, the interplay among on-site Coulomb interaction $U$, superconducting gap $\Delta$, and hybridization $\Gamma_{\mathrm{S} / \mathrm{N}}$ with the superconducting/normal contact determines the nature of the ground state (GS). When $\Delta \rightarrow \infty$ and $\Gamma_{N}=0$, there are no quasiparticles, so only subgap excitations are possible. A superconducting pair correlation then enters into the effective Hamiltonian as an on-site term whose strength is proportional to $\Gamma_{S}$. Then the Hamiltonian can be easily diagonalized [20-22]. Singlet $|S\rangle$ and doublet $|D\rangle$ GSs are possible depending on the relative strengths of $U$ and $\Gamma_{S}$. When

$$
E_{A} \equiv \sqrt{\left(\varepsilon_{d}+U / 2\right)^{2}+\Gamma_{S}^{2}}>U / 2,
$$

where $\varepsilon_{d}$ denotes the dot's energy level, a singlet GS is preferred as being the lowest energy state, which is a BCStype combination of empty and doubly occupied dot states. On the other hand, for the opposite case $E_{A}<U / 2$, the GS becomes a doublet (singly occupied dot) state. The phase boundary between singlet and doublet states is determined by the equation $E_{A}=U / 2$ and even-odd parity crossing happens along this boundary [23-26]. For a finite $\Delta$, but till with $\Gamma_{N}=0$, the previous picture is altered due to the presence of available quasiparticles in $S$ contact. When $U \gg \Delta$ but $\Delta>k_{B} T_{K}$ (here $T_{K}$ represents the Kondo binding energy, and $k_{B}$ is the Boltzmann constant), the dot is singly occupied and the localized spin is poorly screened by the lack of the quasiparticles. Here, the GS is the $|D\rangle$. Excitations on top of this GS are termed Yu-Shiba-Rusinov (YSR) bound states
[27] and consist of a singlet state formed by a quasiparticle in the superconductor that is bounded to the dot spin. On the opposite regime, $\Delta<k_{B} T_{K}$ but still $U \gg \Delta$ (by enhancing, for example, $\Gamma_{S}$ ) the GS is a Kondo singlet state, that must be distinguished from the BCS-type singlet mentioned above $[24,28]$. Importantly, the GS parity has changed from doublet to singlet as $\Gamma_{S}$ enhances. On the other hand, for the weak interacting case when $U \ll \Delta$, the superconducting proximity effect on the dot leads to a BCS-type GS in which subgap excitations appear as pairs of YSR states, i.e., superconducting quasiparticles bound antiferromagnetically to the local spin [29-34]. It is worth mentioning that YSR states are regarded as precursors of Majorana states as demonstrated in atomic chains deposited on a superconducting surface [35-45]. A sequence of YSR bound states hybridize along the chain and a band is formed that exhibits a localized density of states just at the ends of the chain. When a normal contact is introduced (i.e., $\Gamma_{N} \neq 0$ ) $[5,17,18,23,29,46-49]$ in the superconducting QD, YSR excitations can coexist with the Kondo resonance formed by the dot electron and normal conduction electrons. As a consequence, the sharp phase boundary between singlet and doublet states is replaced by a smooth crossover between them even if the normal contact is weakly coupled [48].

We address the subgap transport properties for a N-QD-S device in two regimes, namely, the Coulomb blockade (CB) regime and the Kondo regime. The subgap spectrum is accessible through the nonlinear conductance as demonstrated experimentally: $\mathcal{G}=d I / d V_{\mathrm{dc}}$ [13-15]. We first investigate the Coulomb blockade regime and analyze the validity of the Hartree-Fock (HF) approximation that fails to describe this transport regime. We propose a more elaborated decoupling procedure, the so-called Hubbard-I approximation that reproduces the Coulomb blockade in the absence of superconductivity. We generalize such approach to the superconducting case. We report the appearance of anticrossings around the degenerate mean field points, i.e., $\varepsilon_{d \sigma}=-U, 0$ in the subgap spectrum for the superconducting case. The Andreev bound states (ABS) never touch the particle-hole symmetry point and 
their distance enhances with $\Gamma_{S}$. We explain such observation based on the parity conservation with the dot gate position. Second, we address the Kondo regime. Here the $|S\rangle \longleftrightarrow|D\rangle$ transitions occur at the particle-hole symmetry points as parity crossings in the subgap spectrum. Some works have employed the mean-field decoupling to describe such a transition and the occurrence of parity crossings [14,21,26] in hybrid setups. However, such an approach is unable to describe dynamical spin fluctuations that build the many-body Kondo state. As an alternative, we propose to employ the numerical renormalization group (NRG) approach. We compare our results with those reported in the experiment in Ref. [14], showing an excellent agreement. Additionally, in this regime, we show how to characterize the nature of the GS in the Kondo regime when a Zeeman field is applied to the QD.

\section{THEORETICAL DESCRIPTION AND CURRENT EXPRESSION}

We investigate a normal metal-QD-BCS superconductor hybrid setup. The system consists of four terms $\mathcal{H}=\mathcal{H}_{N}+$ $\mathcal{H}_{S}+\mathcal{H}_{D}+\mathcal{H}_{T}$, where

$$
\mathcal{H}_{N}=\sum_{k, \sigma} \varepsilon_{N k} c_{N k \sigma}^{\dagger} c_{N k \sigma}
$$

describes the normal contact with $c_{N k \sigma}^{\dagger}\left(c_{N k \sigma}\right)$ creating (annihilating) an electron of energy $\varepsilon_{N k}$ with wave number $k$ and spin $\sigma=\uparrow / \downarrow$. The BCS-contact Hamiltonian reads

$$
\mathcal{H}_{S}=\sum_{k, \sigma} \xi_{S k} c_{S k \sigma}^{\dagger} c_{S k \sigma}+\sum_{k}\left(\Delta c_{S k \uparrow}^{\dagger} c_{S \bar{k} \downarrow}^{\dagger}+\text { H.c. }\right),
$$

with $c_{S k \sigma}^{\dagger}\left(c_{S k \sigma}\right)$ as the creation (annihilation) operator and $\bar{k}=-k$. The single-particle energy and gap strength in the $S$ contact are denoted by $\xi_{S k}=\varepsilon_{S k}-\mu_{S}$ and $\Delta$, respectively. Since the number of electrons is not conserved due to pair creation (and annihilation) processes in a superconductor, we introduce the chemical potential $\mu_{S}$ in $\mathcal{H}_{S}$ to fix the electron density. Hereafter, we set $\mu_{S}=0$ and only change the chemical potential $\mu_{N}$ of the normal contact, thus the applied bias being $\mathrm{eV}_{\mathrm{dc}}=\mu_{N}$ (the Fermi energy $\left.E_{F}=0\right)$. The single-level dot Hamitonian reads

$$
\mathcal{H}_{D}=\sum_{\sigma} \varepsilon_{d \sigma} d_{\sigma}^{\dagger} d_{\sigma}+U n_{d \uparrow} n_{d \downarrow}
$$

where $\varepsilon_{d \sigma}$ is the dot energy level that in the presence of a magnetic field $B$ reads $\varepsilon_{d \sigma}=\varepsilon_{d}+\sigma E_{Z} / 2$ with $E_{Z}=\mu_{B} g B$ as the Zeeman energy. $d_{\sigma}^{\dagger}\left(d_{\sigma}\right)$ creates (annihilates) electrons at the dot site and $n_{d \sigma}=d_{\sigma}^{\dagger} d_{\sigma}$ is the occupation operator. Finally, the tunneling processes between dot and contacts are represented by

$$
\mathcal{H}_{T}=\sum_{\alpha=N / S, k, \sigma}\left(V_{\alpha} c_{\alpha k \sigma}^{\dagger} d_{\sigma}+\text { H.c. }\right) .
$$

The hybridization strength is characterized by $\Gamma_{\alpha}=$ $\pi \rho_{\alpha}(0) V_{\alpha}^{2}$, where $\rho_{\alpha}(0)$ is the density of states at the Fermi energy of contact $\alpha$ in the normal phase. We focus on the regime $\Gamma_{S} \gg \Gamma_{N}$ and consider the normal contact acting as a probe.

We are interested in solving $\mathcal{H}$ to obtain the energy spectrum. For that purpose, we obtain the poles of the Green's function as

$$
\Re\left(\omega \mathbb{I}-\mathcal{H}+i 0^{+}\right)=0
$$

that give the ABS. Besides, such subgap spectrum is obtained experimentally by measuring the differential conductance $\mathcal{G}=d I / d V_{\mathrm{dc}}$ where $I$ is the electrical current when a $V_{\mathrm{dc}}$ bias voltage is applied between the contacts. Then, we compute the electrical current in this setup but discarding the current originated from the superconducting quasiparticle density of states. Therefore, we calculate the charge current generated solely by the Andreev reflection,

$$
I=\frac{8 e}{h} \int d \varepsilon \Gamma_{N}^{2}\left|\mathcal{G}_{d, d ; 12}^{r}(\varepsilon)\right|^{2}\left[f\left(\varepsilon-\mu_{N}\right)-f\left(\varepsilon+\mu_{N}\right)\right],
$$

where $\mathcal{G}_{d, d ; 12}^{r}(\varepsilon)$ is the retarded anomalous Green's function that describes the conversion of an electron into a hole caused by the scattering on the Bose-Einstein condensed Cooper pairs, i.e., the Andreev-type scattering. Hereafter, the bold symbols denote matrices so the subscripts denote their matrix components. $f(\varepsilon)=1 /\left(e^{\beta \varepsilon}+1\right)$ is the Fermi-Dirac distribution function with $\beta=1 / k_{B} T$. To grasp the underlying physics, we calculate the nonequilibrium Keldysh Green's functions and then $\mathcal{G}_{d, d ; 12}^{r}(\varepsilon)$. For such a purpose, we introduce the Nambu spinor representation defining $\hat{d}=\left(d_{\uparrow}^{\dagger}, d_{\downarrow}\right)$, and $\hat{c}_{\alpha k}=\left(c_{\alpha k \uparrow}^{\dagger}, c_{\alpha \bar{k} \downarrow}\right)$. The Keldysh time-ordered Green's function then takes the form

$$
\mathcal{G}_{A, B}\left(t, t^{\prime}\right) \equiv\left\langle\left\langle\hat{A}(t), \hat{B}^{\dagger}\left(t^{\prime}\right)\right\rangle\right\rangle=-i\left\langle\mathcal{T} \hat{A}(t) \otimes \hat{B}^{\dagger}\left(t^{\prime}\right)\right\rangle,
$$

where $\hat{A}$ and $\hat{B}$ can be any spinor like $\hat{d}$ or $\hat{c}_{\alpha k}$ and $\mathcal{T}$ is the time-ordering operator. The retarded/advanced and lesser Green's functions follow from analytic continuation [50]. In the next sections, we derive the anomalous Green's function for the two regimes of interest, the Coulomb blockade and Kondo regimes.

\section{COULOMB BLOCKADE REGIME}

Here we develop the equation-of-motion (EOM) technique to describe the Coulomb blockade regime. We first analyze the HF approximation that fails to describe this transport regime. Then, we propose to employ a more sophisticated decoupling procedure, the Hubbard-I approximation that reproduces the Coulomb blockade phenomenon for a N-QD system and predicts the occurrence of anticrossings around the degenerate mean-field points, i.e., $\varepsilon_{d \sigma}=-U, 0$ in the subgap spectrum for the superconducting case. The EOM procedure reads

$$
d\langle A(t)\rangle / d t=(i / \hbar)\langle[\mathcal{H}, A(t)]\rangle .
$$

In particular, the time-ordered Green's functions for the dot read

$$
\begin{aligned}
\mathcal{G}_{d, d}\left(t, t^{\prime}\right)= & \mathbf{g}_{d}\left(t, t^{\prime}\right)+\int \frac{d t_{1}}{\hbar} \mathbf{g}_{d}\left(t, t_{1}\right) \mathbf{U D}\left(t_{1}, t^{\prime}\right) \\
& +\int \frac{d t_{1}}{\hbar} \int \frac{d t_{2}}{\hbar} \mathbf{g}_{d}\left(t, t_{1}\right) \boldsymbol{\Sigma}_{0}\left(t_{1}, t_{2}\right) \mathcal{G}_{d, d}\left(t_{2}, t^{\prime}\right),
\end{aligned}
$$

where $\mathbf{U} \equiv U \sigma_{\mathbf{3}}$ and $\mathbf{V}_{\alpha} \equiv V_{\alpha} \sigma_{\mathbf{3}} \quad\left(\sigma_{i}\right.$ with $i=1,2,3$ are the Pauli matrices and $\sigma_{0}$ the $2 \times 2$ identity matrix) $\mathbf{U} \equiv$ 
$\operatorname{diag}(U,-U)$, and $\mathbf{V}_{\alpha} \equiv \operatorname{diag}\left(V_{\alpha},-V_{\alpha}\right)$, and the hopping selfenergy:

$$
\boldsymbol{\Sigma}_{0}\left(t_{1}, t_{2}\right)=\sum_{\alpha, k} \mathbf{V}_{\alpha} \mathbf{g}_{\alpha k}\left(t_{1}, t_{2}\right) \mathbf{V}_{\alpha}=\sum_{\alpha} \boldsymbol{\Sigma}_{0 \alpha}\left(t_{1}, t_{2}\right) .
$$

Here $\mathbf{g}_{d}\left(t, t^{\prime}\right)$ and $\mathbf{g}_{\alpha k}\left(t, t^{\prime}\right)$ denote the noninteracting dot and contact's isolated Green's functions, respectively. In the HF decoupling scheme, the Green's function $\mathbf{D}$ is approximated as

$$
\mathbf{D} \equiv\left\langle\left\langle\left(\begin{array}{c}
d_{\uparrow} n_{d \downarrow} \\
d_{\downarrow}^{\dagger} n_{d \uparrow}
\end{array}\right), \hat{d}^{\dagger}\right\rangle\right) \approx \boldsymbol{\Lambda} \mathcal{G}_{d, d},
$$

where

$$
\boldsymbol{\Lambda}=\left(\begin{array}{cc}
\left\langle n_{d \downarrow}\right\rangle & \mathcal{F} \\
-\mathcal{F}^{\dagger} & \left\langle n_{d \uparrow}\right\rangle
\end{array}\right) .
$$

Here $\mathcal{F}^{\dagger}$ is the anomalous propagator in the Nambu description:

$$
\mathcal{F}^{\dagger}=\left\langle d_{\uparrow}^{\dagger} d_{\downarrow}^{\dagger}\right\rangle .
$$

In the frequency domain, the retarded HF dot Green's function is written as

$$
\mathcal{G}_{d, d}^{r}(\varepsilon)=\left[\mathbf{g}_{d}^{r,-1}(\varepsilon)-\boldsymbol{\Sigma}_{H F}-\boldsymbol{\Sigma}_{0}^{r}(\varepsilon)\right]^{-1} .
$$

Here,

$$
\mathbf{g}_{d}^{r,-1}(\varepsilon)=\operatorname{diag}\left(\varepsilon-\varepsilon_{d \uparrow}+i 0^{+}, \varepsilon+\varepsilon_{d \downarrow}+i 0^{+}\right)
$$

is the unperturbed dot Green's function. The self-energies are, namely, (i) the HF interacting self-energy

$$
\boldsymbol{\Sigma}_{H F}=U \boldsymbol{\Lambda},
$$

and (ii) the hopping self-energy $\boldsymbol{\Sigma}_{0}^{r}(\varepsilon)=\boldsymbol{\Sigma}_{0 S}^{r}(\varepsilon)+\boldsymbol{\Sigma}_{0 N}^{r}(\varepsilon)$ composed by the normal

$$
\mathbf{\Sigma}_{0 N}^{r}(\varepsilon)=-i \Gamma_{N} \sigma_{\mathbf{0}}
$$

and the superconductor contributions,

$$
\boldsymbol{\Sigma}_{0 S}^{r}(\varepsilon)=-i \Gamma_{S}\left[\beta_{d}(\varepsilon) \sigma_{0}+i \sigma_{3} \sigma_{2} \beta_{o}(\varepsilon)\right],
$$

with

$$
\beta_{d}(\varepsilon)=\frac{\Theta(|\varepsilon|-\Delta)|\varepsilon|}{\sqrt{\varepsilon^{2}-\Delta^{2}}}-i \frac{\Theta(\Delta-|\varepsilon|) \varepsilon}{\sqrt{\Delta^{2}-\varepsilon^{2}}}
$$

and

$$
\beta_{o}(\varepsilon)=\frac{\Theta(|\varepsilon|-\Delta) \operatorname{sgn}(\varepsilon) \Delta}{\sqrt{\varepsilon^{2}-\Delta^{2}}}-i \frac{\Theta(\Delta-|\varepsilon|) \Delta}{\sqrt{\Delta^{2}-\varepsilon^{2}}} .
$$

Here, $\Theta(x)$ is the Heaviside function. Finally, the QD mean occupations are evaluated from the lesser Green's function via the relation

$$
\left(\begin{array}{cc}
\left\langle n_{d \uparrow}\right\rangle & \mathcal{F} \\
\mathcal{F}^{\dagger} & 1-\left\langle n_{d \downarrow}\right\rangle
\end{array}\right)=\frac{1}{2 \pi i} \int d \varepsilon \mathcal{G}_{d, d}^{<}(\varepsilon),
$$

where $\mathcal{G}_{d, d}^{<}(\varepsilon)$ corresponds to the lesser HF dot Green's function,

$$
\mathcal{G}_{d, d}^{<}(\varepsilon)=\mathcal{G}_{d, d}^{r}(\varepsilon) \boldsymbol{\Sigma}_{0}^{<}(\varepsilon) \mathcal{G}_{d, d}^{a}(\varepsilon),
$$

with $\boldsymbol{\Sigma}_{0}^{<}(\varepsilon)=\boldsymbol{\Sigma}_{0 N}^{<}(\varepsilon)+\boldsymbol{\Sigma}_{0 S}^{<}(\varepsilon)$ being lesser hopping selfenergy. This self-energy contains the contribution due to the normal lead,

$$
\boldsymbol{\Sigma}_{0 N}^{<}(\varepsilon)=2 i \Gamma_{N} \operatorname{diag}\left(f\left(\varepsilon-\mu_{N}\right), \quad f\left(\varepsilon+\mu_{N}\right)\right),
$$

and the superconducting one,

$$
\begin{aligned}
\Sigma_{0 S}^{<}(\varepsilon)= & \frac{2 i \Gamma_{S} f(\varepsilon) \Theta(|\varepsilon|-\Delta)|\varepsilon|}{\sqrt{\varepsilon^{2}-\Delta^{2}}} \operatorname{diag}(1,1) \\
& +\frac{\Delta}{\varepsilon} \overline{\operatorname{diag}}(-1,-1),
\end{aligned}
$$

where $\overline{\operatorname{diag}}($,$) denotes the antidiagonal of a matrix. The$ advanced Green's function is obtained from the relation $\mathcal{G}_{d, d}^{a}(\varepsilon)=\left[\mathcal{G}_{d, d}^{r}(\varepsilon)\right]^{\dagger}$. Equation (22) now has to be solved self-consistently since in both sides of the equality the dot's average occupations appear.

In the following, we analyze the weakness of the HF approximation to treat both the Coulomb blockade and Kondo regime. Such an approach has been proposed to deal with Kondo physics since it reproduces some of the features of the Kondo-like ABS spectrum. However, as we indicate below, such characteristics are related to symmetry-broken states that in the end should be averaged. In that respect, HF fails to explain properly the underlying physics in the Kondo regime. Besides, we show how the HF approach fails in describing the Coulomb blockade regime for which a suitable approach within the EOM scheme is proposed.

To gain a better understanding of the HF approximation, we analyze why such an approach has been proposed to explain Kondo physics. We first consider $\Gamma_{N}=0$ and $\Delta=0$. The solution for $\left\langle n_{d \uparrow}\right\rangle,\left\langle n_{d \downarrow}\right\rangle$ from Eq. (22) can be encountered in terms of

$$
n=\left\langle n_{d \uparrow}\right\rangle+\left\langle n_{d \downarrow}\right\rangle,
$$

the total occupation, and

$$
m=\left\langle n_{d \uparrow}\right\rangle-\left\langle n_{d \downarrow}\right\rangle,
$$

the magnetization. According to Anderson's idea [51], in the HF approximation there are two possible solutions for $n$ and $m$ : a nonmagnetic solution with $m=0$ and a magnetic $m \neq 0$ solution. A simple analysis at zero temperature shows that

$$
\begin{aligned}
& n=\frac{1}{\pi} \sum_{\sigma} \cot ^{-1}\left(\frac{\varepsilon_{d}+U(n-\sigma m) / 2}{\Gamma_{S}}\right), \\
& m=\frac{1}{\pi} \sum_{\sigma} \sigma \cot ^{-1}\left(\frac{\varepsilon_{d}+U(n-\sigma m) / 2}{\Gamma_{S}}\right) .
\end{aligned}
$$

To find the phase boundary between magnetic and nonmagnetic regions, we set $m \rightarrow 0^{+}$and expand Eqs. (28) to get

$$
\begin{aligned}
n & \approx \frac{2}{\pi} \cot ^{-1}\left(\frac{\varepsilon_{d}+U n / 2}{\Gamma_{S}}\right), \\
m & \approx \frac{U m}{\pi \Gamma_{S}} \sin ^{2}(\pi n / 2) .
\end{aligned}
$$

For $m \neq 0$, Eq. (29b) allows a solution only when $U / \pi \Gamma_{S}>1$ so it is a necessary condition to obtain a magnetic solution. In our HF calculations with $\Delta \neq 0$, we consider $U=2.5 \Delta$ and $\Gamma_{S}=0.2 \Delta$ and thus $U / \pi \Gamma_{S}>1$ is fulfilled. In 


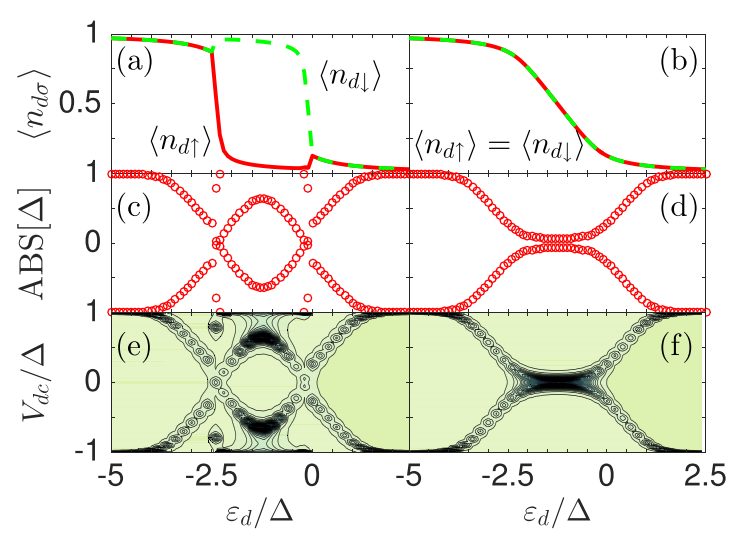

FIG. 1. (a) Dot's average occupation $\left\langle n_{d \sigma}\right\rangle$ for $m \neq 0$ and (b) for $m=0$. (c) Andreev bound states (ABS) for $m \neq 0$ and (d) for $m=0$. The differential conductance $d I / d V_{\mathrm{dc}}$ as a function of the gate $\varepsilon_{d}$ in (e) for $m \neq 0$ and (f) for $m 0$. Dark areas in (e) and (f) indicate high $d I / d V_{\mathrm{dc}}$. Calculations for $m=0$ are performed by the restricted Hartree-Fock method whereas for $m \neq 0$ the unrestricted Hartree-Fock approach was employed. Parameters: $\Delta=1, E_{Z} / \Delta=$ $0, U / \Delta=2.5, \Gamma_{S} / \Delta=0.2, \Gamma_{N} / \Gamma_{S}=1 / 3, \beta / \Delta=500$.

the literature, the case with $m \neq 0$ is denominated unrestricted Hartree-Fock (UHF) while the restricted Hartree-Fock (RHF) means the solution with $m=0$. The peculiarity of the UHF is that it breaks spin-reversal symmetry, although the Hamiltonian $\mathcal{H}$ is invariant under such symmetry. Besides, whenever it is possible, a $m>0$ solution, there is always a corresponding degenerate negative $-m$ solution. The breakdown of the spinreversal symmetry of the UHF can be cured by averaging twodegenerate solutions at the very end [52]. To emphasize the effect of the magnetic solution, we do not average and show in Fig. 1(a) the spin $\uparrow$ and spin $\downarrow$ occupations, namely, $\left\langle n_{d \uparrow}\right\rangle$, and $\left\langle n_{d \downarrow}\right\rangle$ versus $\varepsilon_{d \sigma}=\varepsilon_{d}$ (we set $E_{Z}=0$ unless otherwise stated). The popularity of such an approach resides in its use to describe the Kondo physics in the ABS spectrum. We recall that $\mathrm{ABS}$ are found by examining the poles of the retarded Green's function:

$$
\left[\Re\left(\mathbf{g}_{d}^{r,-1}(\varepsilon)-\boldsymbol{\Sigma}_{H F}-\Sigma_{0}^{r}(\varepsilon)\right)\right]=0 .
$$

For the hybrid setup, the presence of the $\mathrm{N}$ contact not only broadens the $\mathrm{ABS}$ as they acquire a finite lifetime but it could enhance the spin exchange interactions and induced the subgap Kondo effect upon approaching to the quantum phase transition which indeed replaced by a crossover [48,53]. Since we are interested in the ABS positions and $\Gamma_{N} \ll \Gamma_{S}$ we discard in the next calculations normal contact. In Fig. 1(c), we see that the positions of the ABS are probed by the the nonlinear conductance plotted in Fig. 1(e). In Figs. 1(c) and 1(e), we see that the positions of the ABS are closely related with the dot's average occupations. When the dot's occupation changes sharply then the ABS localize near the Fermi level, while they move near the gap edges $\pm \Delta$ when the dot occupation is kept fixed. As we see in Fig. 1(c) and 1(e) the ABS display a loop structure that indeed represents the quantum phase transition from a singlet to a doublet GS as reported experimentally $[13,14]$ for the Kondo regime. This is because the UHF approach implies a local magnetic moment solution $(m \neq 0)$. The exchange interaction between conduction electrons and a local magnetic moment gives rise to the YSR subgap excitations inside the superconducting gap [27]. The remaining question is what is the strength of the local exchange energy $E_{e x}$ with the UHF. In the presence of a local moment, $\mathcal{H}_{D}$ can be replaced by a noninteracting single site Hamiltonian with $\varepsilon_{d \uparrow}=\varepsilon_{d}+E_{\mathrm{ex}}$ and $\varepsilon_{d \downarrow}=\varepsilon_{d}-$ $E_{\text {ex }}$ where $E_{\text {ex }}=U\left(\left\langle n_{d \downarrow}\right\rangle-\left\langle n_{d \uparrow}\right\rangle\right) / 2[21]$.

The UHF solution well describes the physics which results from the existence of a local magnetic moment and for such reason it reproduces the spectrum in the Kondo regime. However, the magnetic moment which is found in the UHF scheme is a static moment, but not a dynamic one. This indicates that the moment in the UHF is not dynamically screened by the conduction electrons and therefore it cannot explain the Kondo physics.

In the next section, we show that HF approximation also fails in describing the Coulomb blockade regime. To illustrate this, we have plotted in Figs. 1(b), 1(d) and 1(f) our results for the occupations, $\mathrm{ABS}$, and $\mathcal{G}$, respectively, when the $m$ and $-m$ solutions are averaged (the RHF approach). We observe that now $\left\langle n_{d \uparrow}\right\rangle=\left\langle n_{d \downarrow}\right\rangle$ whereas the ABS and $\mathcal{G}$ do not display the looplike structure obtained in the UHF approximation. Indeed, it is known that the RHF approach leads to a single resonance peak centered at an effective dot-level position renormalized by $U$, which is contradictory with the physics in the Coulomb blockade regime where two resonance peaks are observed due to the quantization of the dot's average occupancy at low enough temperatures. Therefore, to describe the $\mathrm{CB}$ regime properly, we implement a different truncation scheme in the EOM procedure. We consider the equation of motion for $\mathbf{D}$ together with Eq. (10) and get

$$
\begin{aligned}
\mathbf{D}\left(t, t^{\prime}\right)= & \mathbf{g}_{d U}\left(t, t^{\prime}\right) \mathbf{n}_{d} \\
& +\sum_{\alpha, k} \int \frac{d t_{1}}{\hbar} \mathbf{g}_{d U}\left(t, t_{1}\right) \mathbf{n}_{d} \mathbf{V}_{\alpha} \mathcal{G}_{\alpha k, d}\left(t_{1}, t^{\prime}\right),
\end{aligned}
$$

where $\mathbf{n}_{d}=\operatorname{diag}\left(\left\langle n_{d \downarrow}\right\rangle,\left\langle n_{d \uparrow}\right\rangle\right)$ and

$$
\mathbf{g}_{d U}^{r,-1}(\varepsilon)=\left(\begin{array}{ll}
\varepsilon-\varepsilon_{d \uparrow}-U+i 0^{+} & \\
& \varepsilon+\varepsilon_{d \downarrow}+U+i 0^{+}
\end{array}\right) .
$$

In obtaining Eq. (31), we made the following decoupling:

$$
\left\langle\left\langle c_{\alpha k \uparrow} n_{d \downarrow}, d_{\uparrow}^{\dagger} / d_{\downarrow}\right\rangle\right\rangle \approx\left\langle n_{d \downarrow}\right\rangle\left\langle\left\langle c_{\alpha k \uparrow}, d_{\uparrow}^{\dagger} / d_{\downarrow}\right\rangle\right\rangle
$$

and

$$
\left\langle\left\langle c_{\alpha k \downarrow}^{\dagger} n_{d \uparrow}, d_{\uparrow}^{\dagger} / d_{\downarrow}\right\rangle\right\rangle \approx\left\langle n_{d \uparrow}\right\rangle\left\langle\left\langle c_{\alpha k \downarrow}^{\dagger}, d_{\uparrow}^{\dagger} / d_{\downarrow}\right\rangle\right\rangle,
$$

and neglected the other generated higher-order Green's functions. Substituting Eq. (31) into Eq. (10) and doing little algebra, we find

$$
\begin{aligned}
\mathcal{G}_{d, d}^{r}(\varepsilon)= & {\left[\mathbf{g}_{d}^{r,-1}(\varepsilon)-\boldsymbol{\Sigma}_{0}^{r}(\varepsilon)-\mathbf{U g}_{d U}^{r}(\varepsilon) \mathbf{n}_{d} \boldsymbol{\Sigma}_{0}^{r}(\varepsilon)\right]^{-1} } \\
& \times\left[1+\mathbf{U g}_{d U}^{r}(\varepsilon) \mathbf{n}_{d}\right] .
\end{aligned}
$$

The isolated dot Green's functions $\mathbf{g}_{d}^{r}(\varepsilon)$ and $\mathbf{g}_{d U}^{r}(\varepsilon)$ have poles close to $\approx \varepsilon_{d \sigma}$ and $\approx \varepsilon_{d \sigma}+U$, and hence we expect that Eq. (35) will show two resonance peaks. Again we employ Eq. (23) to obtain the QD average occupations. In Fig. 2(a), we show $\left\langle n_{d \sigma}\right\rangle$ versus $\varepsilon_{d}$. It displays the well-defined plateaus of width $U$. The ABS are obtained from the poles of the 


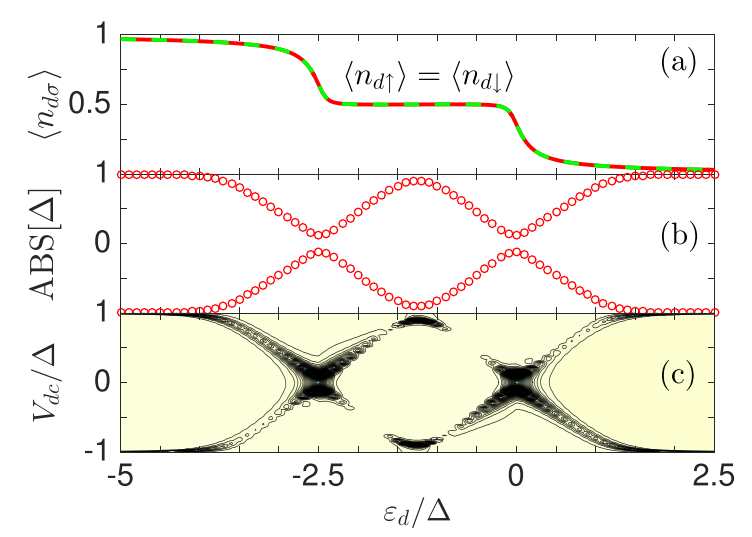

FIG. 2. (a) Dot's average occupation $\left\langle n_{d \sigma}\right\rangle$, (b) Andreev bound states (ABS), and (c) differential conductance $d I / d V_{d c}$ as a function of the gate $\varepsilon_{d}$ in the Coulomb-blockade regime. Parameters: $\Delta=1$, $U / \Delta=2.5, \Gamma_{S} / \Delta=0.2, \Gamma_{N} / \Gamma_{S}=1 / 3, \beta / \Delta=500$.

determinant:

$$
\left[\Re\left(\mathbf{g}_{d}^{r,-1}(\varepsilon)-\boldsymbol{\Sigma}_{0}^{r}(\varepsilon)-\mathbf{U g}_{d U}^{r}(\varepsilon) \mathbf{n}_{d} \boldsymbol{\Sigma}_{0}^{r}(\varepsilon)\right)\right]^{-1}=0 .
$$

The loop structure is observed in the singly occupied region as shown in Fig. 2(b). Importantly, the ABS never cross at the degenerate points due to the absence of spin fluctuations in the $\mathrm{CB}$ regime. The evolution of the loop structure with $\Gamma_{S}$ is shown in Fig. 3. The loop height becomes smaller when we increase $\Gamma_{S}$ and completely disappears when $\Gamma_{S} \sim U$. Besides, ABS never touches the particle-hole symmetry point even if $\Gamma_{S}$ increases. In the $\mathrm{CB}$ regime, the singly occupied dot is not screened by conduction electrons, so we cannot expect the transition from doublet to Kondo screened singlet. Instead, as $\Gamma_{S}$ is increased, a possible scenario is the appearance of the BCS-type singlet which consists of $|0\rangle$ (empty) and $|\uparrow \downarrow\rangle$ (doubly occupied) dot states. Hence, the crossing of the ABS at the particle-hole symmetric point is not expected even when $\Gamma_{S}$ increases. Note that a further increase of $\Gamma_{S}$ would induce the appearance of Kondo correlations that are not included in the present description. For that purpose, we analyze in the next section the Kondo regime by employing the NRG

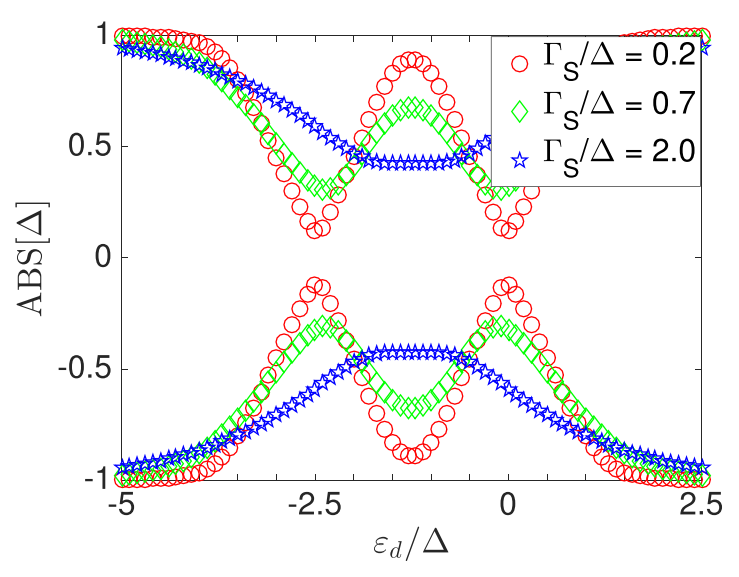

FIG. 3. Andreev bound states (ABS) as a function of the gate $\varepsilon_{d}$ in the Coulomb-blockade regime for various $\Gamma_{S}$. Parameters: $\Delta=1$, $E_{Z} / \Delta=0, U / \Delta=2.5, \Gamma_{S} / \Delta=0.2, \Gamma_{N} / \Gamma_{S}=1 / 3, \beta / \Delta=500$.

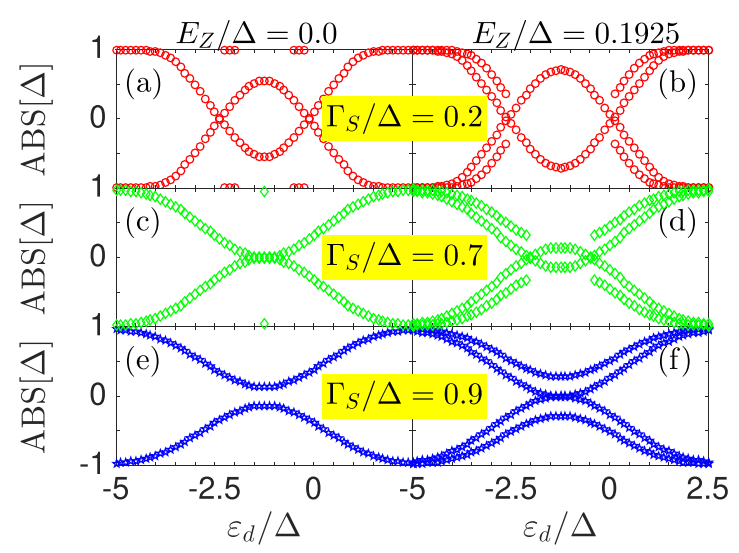

FIG. 4. NRG Subgap spectrum for different $\Gamma_{S}$ values at zero and finite magnetic field. $E_{Z}=0$ (a) shows the ABS as a function of $\varepsilon_{d}$ for $\Gamma_{S} / \Delta=0.2$, (c) for $\Gamma_{S} / \Delta=0.7$, and (e) for $\Gamma_{S} / \Delta=0.9$. In the presence of magnetic field $E_{Z} / \Delta=0.1935$, the Andreev subgap spectrum is shown in (b) for $\Gamma_{S} / \Delta=0.2$, (d) for $\Gamma_{S} / \Delta=0.7$, and (f) for $\Gamma_{S} / \Delta=0.9$. Other parameters: $D=1$ (half bandwidth), $\Delta=$ $0.04, U / \Delta=2.5, \Gamma_{N} / \Gamma_{S}=0$.

calculation which is a sophisticated numerical technique that produces quite accurate results.

\section{KONDO REGIME}

We investigate the regime in which the Kondo singlet becomes relevant. Experimentally, this situation has been explored in hybrid QDs in Ref. [14]. There, the subgap spectrum was measured using the nonlinear conductance $\mathcal{G}$. The experiment reported the observation of the $|S\rangle \longleftrightarrow|D\rangle$ transition and parity crossings from the Andreev spectrum. Here, we reproduce those results by employing the standard NRG method [54]. For the ABS analysis, we consider the QD connected to the superconducting contact $\left(\Gamma_{N}=0\right)$. We are aware of the role of the normal contact by enhancing the spinexchange interactions and then inducing the subgap Kondo formation that replaces the quantum phase transition by a crossover [48,53]. However, for the considered parameters, $\Gamma_{N}$ acts as a probe and our calcualtions for the S-QD system reproduce the experimental findings [14]. The underlying physics is determined by the interplay between singlet and doublet states. Here, we consider the experimental situation of Ref. [14]: $U / \Delta=2.5, D=1$ (half bandwidth) and $\Delta=$ $0.04 D$ at zero temperature. For different $\Gamma_{S}$, we observe a loop structure with crossings at the degenerate mean-field points. As discussed before such loop structure is also observed in Fig. 1(c) when the UHF approach was used. However, we pointed out that indeed the UHF approach fails to describe a dynamical spin flip for the Kondo-like impurity and instead it offers a static spin screening. Besides, whereas the UHF spectrum presents a divergent behavior at the degenerate points, the NRG calculation gives true crossing points. Furthermore, the NRG spectrum exhibits two subgap excitations around the doublet-singlet or singlet-doublet transition points due to the existence of two BCS-like singlet excited states that are not predicted by the HF approximation. As $\Gamma_{S}$ is increased, our findings for Figs. 4(c)-4(e) display the gradual disappearance 


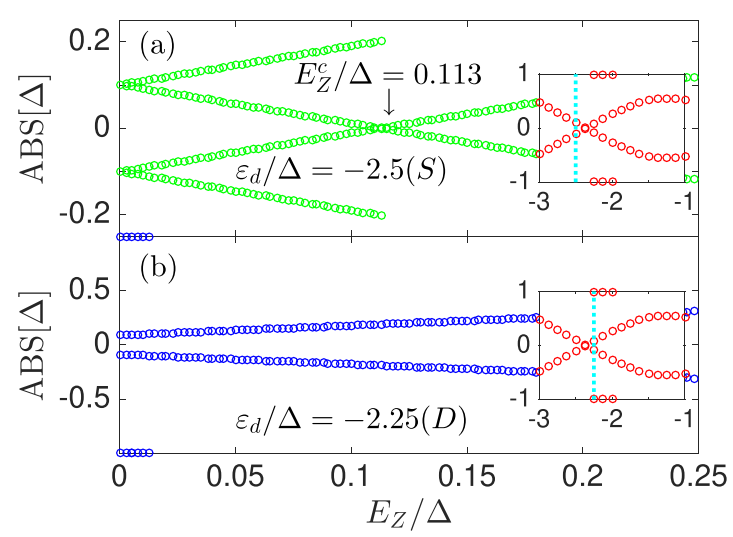

FIG. 5. (a) Subgap spectrum as a function of $E_{Z}$ for $\varepsilon_{d} / \Delta=$ -2.5 . Here, the ground state is a singlet when $E_{Z}=0$. (b) ABS for $\varepsilon_{d} / \Delta=-2.25$. In this case, the ground state is a doublet when $E_{Z}=0$. The insets display magnified plots of Fig. 4 around the singlet-doublet transition. Parameters: $D=1$ (half-bandwidth), $\Delta=$ $0.04, U / \Delta=2.5, \Gamma_{S} / \Delta=0.2, \Gamma_{N} / \Gamma_{S}=0$.

of the loop structure, which means that the GS changes from doublet to singlet states in the singly occupied region (closed at $\varepsilon_{d}=-U / 2$ when the Kondo singlet prevails).

Now we consider the presence of a magnetic field $B$ with an associated Zeeman energy $E_{Z}=g \mu_{B} B$. The application of a Zeeman field, $E_{Z} \neq 0$, unambiguously identifies the GS for the dot [14]. Our results are shown in Figs. 4(b), 4(d) and 4(f). For $E_{Z} \neq 0$, we also observe four ABS in the doubly and empty occupied regions when $\varepsilon_{d} / \Delta<-U / 2$ and $\varepsilon_{d} / \Delta>0$. In this region, the ground sate is a BCS singlet, while the excited state is a doublet. For $E_{Z} \neq 0$, the doublet state is Zeeman split so two transitions are possible from ground to excited states. The transition from a lower-energy singlet state to a higher-energy singlet state is not allowed because this process conserves the fermion parity. In this respect, the magnetic field drives a quantum phase transition as shown in Figs. 4(c) and 4(d). Thus, the four subgap states shown in Figs. 4(d) are excitations from the GS, a BCS singlet state, to the Zeeman-split doublet state.

All the previous features are nicely collected in Fig. 5. Here, the ABS as a function of Zeeman energy $E_{Z}$ are plotted. We focus on the region near the singlet-doublet transition point when $E_{Z}=0$ [see Fig. 4(a) and also insets of Fig. 5]. In the absence of an applied magnetic field, for $\varepsilon_{d} / \Delta=-2.5$ the GS is a singlet so possible excitations are transitions to doublet states. When we apply a magnetic field, the degenerate doublet state splits into two distinct states due to the Zeeman effect, but the singlet GS remains unchanged. Therefore, there are two possible transitions from singlet to doublet states and we get four ABS [see Fig. 5(a)]. At $E_{Z} / \Delta>0.115$, the GS changes from singlet to doublet states and only two ABS are possible. The transition from the lower-energy doublet state to the higher-energy doublet state is not allowed since the fermion parity has to be changed during the transition and hence we get only two ABS. On the other hand, if we look at $\varepsilon_{d} / \Delta=-2.25$ and $E_{Z}=0$, the GS is a doublet. For $E_{Z} \neq 0$ [see Fig. 5(b)], the doublet GS splits, but the transitions between doublet states is not permitted and we obtain two ABS. As a matter of fact, in the presence of the normal contact, the single singlet-doublet phase transition point is replaced by the broad crossover region [48]. Therefore, the presence of a magnetic field allows us to identify unambiguously the nature of the dot GS.

\section{CONCLUSIONS}

In summary, we have calculated the subgap spectrum for two different transport regimes, namely, the Coulomb blockade regime and the Kondo regime. For the Coulomb blockade regime, we have used the equation-of-motion technique and propose a generalization of the the Hubard-I decoupling scheme to deal with superconducting contacts. We have shown that a mean-field approach as the HF approach is unable to describe nor the charge blockade regime neither the Kondo regime. We have found that the subgap Andreev spectrum in the Coulomb blockade regime exhibits anticrossing points around the degenerate mean-field points $\varepsilon_{d}=-U$ and $\varepsilon_{d}=0$. The occurrence of anticrossings signals the conservation of the parity GS against the tuning of the dotlevel position. For $\varepsilon_{d}<-U$, the GS corresponds to a doubly occupied state whereas for $\varepsilon_{d}>0$ the state corresponds to an empty configuration. For $-U \lesssim \varepsilon_{d} \lesssim 0$, the GS is a BCS singlet state with a superposition of empty and doubly dot states. In all the cases, the parity is even.

For the Kondo regime, we have employed the NRG calculation and reproduce the experimental results reported in Ref. [14]. Finally, we have shown that under the application of a magnetic field it is possible to identify the nature of the GS in the subgap spectrum by counting the number of possible excitations. These results match with those found experimentally for the B-field case [14]. We hope that our findings push forward experimental research in the Coulomb blockade transport regime for hybrid NS-QD systems and can confirm the reported subgap features as anticrossings at the degenerate points.

\section{ACKNOWLEDGMENTS}

We are grateful to D. Sánchez for fruitful discussions. We acknowledge the FEDER/MICINN/AEI Grant No. MAT2017-82639 for its financial support.
[1] A. V. Balatsky and I. Vekhter, Jian-Xin Zhu, Rev. Mod. Phys. 78, 373 (2006).

[2] S. De Franceschi, Leo Kouwenhoven, C. Schönenberger, and W. Werns-dorfer, Nat. Nanotechnol. 5, 703 (2010).
[3] A. Yazdani, B. A. Jones, C. P. Lutz, M. F. Crommie, and D. M. Eigler, Science 275, 1767 (1997).

[4] M. R. Buitelaar, W. Belzig, T. Nussbaumer, B. Babić, C. Bruder, and C. Schönenberger, Phys. Rev. Lett. 91, 057005 (2003). 
[5] M. R. Gräber, T. Nussbaumer, W. Belzig, and C. Schönenberger, Nanotechnology 15, S479 (2004).

[6] A. Eichler, M. Weiss, S. Oberholzer, C. Schönenberger, A. Levy Yeyati, J. C. Cuevas, A. Martín-Rodero, Phys. Rev. Lett. 99, 126602 (2007).

[7] K. Grove-Rasmussen, H. I. Jørgensen, B. M. Andersen, J. Paaske, T. S. Jespersen, J. Nygård, K. Flensberg, P. E. Lindelof, Phys. Rev. B 79, 134518 (2009).

[8] J.-D. Pillet, C. H. L. Quay, P. Morfin, C. Bena, A. L. Yeyati, and P. Joyez, Nat. Phys. 6, 965 (2010).

[9] J.-D. Pillet, P. Joyez, R. Žitko, and M. F. Goffman, Phys. Rev. B 88, 045101 (2013).

[10] B.-K. Kim, Y.-H. Ahn, J.-J. Kim, M.-S. Choi, M.-H. Bae, Kicheon Kang, Jong Soo Lim, R. López, and N. Kim, Phys. Rev. Lett. 110, 076803 (2013).

[11] J. Schindele, A. Baumgartner, R. Maurand, M. Weiss, and C. Schönenberger, Phys. Rev. B 89, 045422 (2014).

[12] Y.-J. Doh, S. De Franceschi, E. P. A. M. Bakkers, and L. P. Kouwenhoven, Nano Lett. 8, 4098 (2008).

[13] E. J. H. Lee, X. Jiang, R. Aguado, G. Katsaros, C. M. Lieber, and S. De Franceschi, Phys. Rev. Lett. 109, 186802 (2012)

[14] E. J. H. Lee, X. Jiang, M. Houzet, R. Aguado, C. M. Lieber, and S. De Franceschi, Nat. Nanotechnol. 9, 79 (2014).

[15] S. Li, N. Kang, P. Caroff, and H. Q. Xu, Phys. Rev. B 95, 014515 (2017).

[16] C. Buizert, A. Oiwa, K. Shibata, K. Hirakawa, and S. Tarucha, Phys. Rev. Lett. 99, 136806 (2007).

[17] R. S. Deacon, Y. Tanaka, A. Oiwa, R. Sakano, K. Yoshida, K. Shibata, K. Hirakawa, and S. Tarucha, Phys. Rev. B 81, 121308(R) (2010).

[18] R. S. Deacon, Y. Tanaka, A. Oiwa, R. Sakano, K. Yoshida, K. Shibata, K. Hirakawa, and S. Tarucha, Phys. Rev. Lett. 104, 076805 (2010).

[19] T. Dirks, T. L. Hughes, S. Lal, B. Uchoa, Y.-F. Chen, C. Chialvo, P. M. Goldbart, and N. Mason, Nat. Phys. 7, 386 (2011).

[20] I. Affleck, J.-S. Caux, and A. M. Zagoskin, Phys. Rev. B 62, 1433 (2000).

[21] E. Vecino, A. Martín-Rodero, and A. Levy Yeyati, Phys. Rev. B 68, 035105 (2003).

[22] A. Oguri, Y. Tanaka, and A. C. Hewson, J. Phys. Soc. Jpn. 73, 2494 (2004).

[23] Y. Tanaka, N. Kawakami, and A. Oguri, J. Phys. Soc. Jpn. 76, 074701 (2007).

[24] J. Bauer, A. Oguri, and A. C. Hewson, J. Phys.: Condens. Matter 19, 486211 (2007).

[25] T. Meng, S. Florens, and P. Simon, Phys. Rev. B 79, 224521 (2009).

[26] A. Martín-Rodero and A. Levy Yeyati, J. Phys.: Condens. Matter 24, 385303 (2012).

[27] L. Yu, Acta Phys. Sin. 21, 75 (1965); H. Shiba, Prog. Theor. Phys. 40, 435 (1968); A. I. Rusinov, Zh. Eksp. Teor. Fiz. Pis'ma Red. 9, 146 (1968) [JETP Lett. 9, 85 (1969)].

[28] T. Yoshioka and Y. Ohashi, J. Phys. Soc. Jpn. 69, 1812 (2000).
[29] L. Li, Z. Cao, T.-F. Fang, H.-G. Luo, and W.-Q. Chen, Phys. Rev. B 94, 165144 (2016).

[30] M. T. Deng, S. Vaitiekenas, E. B. Hansen, J. Danon, M. Leijnse, K. Flensberg, and J. Nygård, P. Krogstrup, and C. M. Marcus, Science 354, 1557 (2016).

[31] G. L. R. Anselmetti, E. A. Martinez, G. C. Ménard, D. Puglia, F. K. Malinowski, J. S. Lee, S. Choi, M. Pendharkar, C. J. Palmstrøm, C. M. Marcus, L. Casparis, and A. P. Higginbotham, Phys. Rev. B 100, 205412 (2019).

[32] E. Vernek, P. H. Penteado, A. C. Seridonio, and J. C. Egues, Phys. Rev. B 89, 165314 (2014).

[33] C.-X. Liu, J. D. Sau, T. D. Stanescu, and S. Das Sarma, Phys. Rev. B 96, 075161 (2017); A. Ptok, A. Kobiałka, and T. Domański, ibid. 96, 195430 (2017).

[34] C. Reeg, O. Dmytruk, D. Chevallier, D. Loss, and J. Klinovaja, Phys. Rev. B 98, 245407 (2018)

[35] S. Nadj-Perge, I. K. Drozdov, B. A. Bernevig, Ali Yazdani, Phys. Rev. B 88, 020407(R) (2013).

[36] Y. Peng, F. Pientka, L. I. Glazman, and F. von Oppen, Phys. Rev. Lett. 114, 106801 (2015).

[37] M. Ruby, F. Pientka, Y. Peng, F. von Oppen, B. W. Heinrich, and K. J. Franke, Phys. Rev. Lett. 115, 197204 (2015).

[38] S. R. Elliott and M. Franz, Rev. Mod. Phys. 87, 137 (2015).

[39] Y. Kim, J. Zhang, E. Rossi, and R. M. Lutchyn, Phys. Rev. Lett. 114, 236804 (2015).

[40] F. Pientka, L. I. Glazman, and F. von Oppen, Phys. Rev. B 88, 155420 (2013).

[41] S. Nadj-Perge, and I. Drozdov, K. Li. Jian, H. Chen, S. Jeon, J. Seo, A. H. MacDonald, Bernevig B. Andrei, A. Yazdani, Ali, Science 346, 602 (2014).

[42] B. E. Feldman, M. T. Randeria, J. Li, S. Jeon, Y. Xie, Z. Wang, I. K. Drozdov, Bernevig, B. Andrei, A. Yazdani, Nat. Phys. 13, 286 (2017).

[43] J. Li, S. Jeon, Yonglong Xie, A. Yazdani, and B. A. Bernevig, Phys. Rev. B 97, 125119 (2018).

[44] B. Jäck, Y. Xie, J. Li, Jian, S. Jeon, Bernevig, B. Andrei and A. Yazdani, Science 364, 1255 (2019).

[45] R. Pawlak, M. Kisiel, J. Klinovaja, T. Meier, S. Kawai, T. Glatzel, D. Loss, and E. Meyer, Quantum Information 2, 16035 (2016).

[46] S. Pfaller, A. Donarini, and M. Grifoni, Phys. Rev. B 87, 155439 (2013).

[47] J.B arański and T. Domański, J. Phys.: Condens. Matter 25, 435305 (2013).

[48] R. Žitko, J. S. Lim, R. López, and R. Aguado, Phys. Rev. B 91, 045441 (2015).

[49] A. Jellinggaard, K. Grove-Rasmussen, M. H. Madsen, and J. Nygård, Phys. Rev. B 94, 064520 (2016).

[50] D. C. Langreth and J. W. Wilkins, Phys. Rev. B 6, 3189 (1972).

[51] P. W. Anderson, Phys. Rev. 124, 41 (1961).

[52] D. E. Logan, M. P. Eastwood, and M. A. Tusch, J. Phys.: Condens. Matter 10, 2673 (1998).

[53] T. Domański, I. Weymann, M. Barańska, and G. Górski, Sci. Rep. 6, 23336 (2016).

[54] R. Bulla, T. A. Costi, and T. Pruschke, Rev. Mod. Phys. 80, 395 (2008). 